\title{
Perioperative Risk Factors Related to Systemic Inflammatory Response Syndrome after Percutaneous Nephrolithotomy
}

\author{
Ho Seok Chung, Seung Il Jung \\ Department of Urology, Chonnam National University Medical School, Gwangju, Korea
}

Percutaneous nephrolithotomy (PCNL) has become the standard treatment for large and complex kidney stones since its introduction in 1976. On the other hand, postoperative complications are not rare and can be quite significant. Of these complications, postoperative infectious complications, such as systemic inflammatory response syndrome (SIRS), remain a seminal event and a possible cause of perioperative mortality. Several recent studies have found a number of factors that increase the risk of SIRS after PCNL. These include preoperative factors (patient's characteristics) and factors associated with the intraoperative and postoperative findings. The present study describes the perioperative risk factors of SIRS after PCNL. These findings can help decrease the incidence of infectious complications, including SIRS, in patients undergoing PCNL.

Keywords: Percutaneous nephrolithotomy; Risk factors; Systemic inflammatory response syndrome; Urinary tract infections

Copyright (c) 2019, Korean Association of Urogenital Tract Infection and Inflammation. All rights reserved. (C) (1) This is an open access article distributed under the terms of the Creative Commons Attribution
Ny unrestricted non-commercial use, distribution, and reproduction in any medium, provided the original work is properly cited.
Received: 1 April, 2019

Revised: 23 April, 2019

Accepted: 23 April, 2019

\author{
Correspondence to: Seung Il Jung \\ (iD) https://orcid.org/0000-0003-4864-8175 \\ Department of Urology, Chonnam National \\ University Hwasun Hospital, 322 Seoyang-ro, \\ Hwasun-eup, Hwasun 58128, Korea \\ Tel: +82-61-379-7749, Fax: +82-61-379-7750 \\ E-mail: drjsi@yahoo.co.kr
}

\section{INTRODUCTION}

Since its introduction in 1976, percutaneous nephrolithotomy (PCNL) has been used widely as the standard treatment for large and complex kidney stones [1]. With the ongoing advances in endourological instruments and imaging modalities, PCNL has become a safer and more effective procedure. On the other hand, PCNL is not without the risk of significant complications [2]. Infectious complications, ranging from fever to septic shock, can occur after PCNL [3].

To avoid infection, antibiotic prophylaxis has been recommended as part of the guidelines for patients undergoing PCNL [3-6]. Despite the use of antibiotics, infectious complications leading to sepsis remain the most common cause of perioperative mortality after the procedure [2]. Systemic inflammatory response syndrome (SIRS), which includes fever, tachycardia, tachypnea and leukocytosis, is a frequent complication after PCNL $[7,8]$. If not well controlled in a timely manner, SIRS can lead to sepsis with multiple organ failure that can threaten the patient's life [9]. Therefore, it is important to minimize the risk of infectious complications after PCNL by identifying the perioperative risk factors.

Several studies have examined the potential risk factors for the development of postoperative SIRS in PCNL patients as well as various approaches for preventing this condition [7-10]. This paper describes the current findings with regard to the perioperative risk factors for SIRS after PCNL.

\section{INCIDENCE}

Many studies have reported the incidence of infectious complications after PCNL, but the analysis in those studies 
was complicated by the differences in the applied definitions. The American College of Chest Physicians and the Society of Critical Care Medicine published a joint statement on the definitions of SIRS, infection, and sepsis (Table 1) [11]. SIRS is diagnosed in patients who meet two or more of the clinical findings in the categories listed in Table 1. Most studies use terms for infectious conditions according to these definitions, but a few studies did not conform to these strict definitions.

Several studies reported the incidence of SIRS after PCNL. A study of 209 Chinese patients reported a $23.4 \%$ incidence of SIRS [7]. In other Asian studies, the rates of post-PCNL SIRS range from $10.7 \%$ to $35.3 \%$ [9,12]. Similarly, the reported incidence of SIRS after PCNL in Western countries ranges from $9.8 \%$ to $21.3 \%[10,13-15]$.

\section{RISK FACTORS FOR SIRS AFTER PCNL}

\section{Preoperative Factors}

Several studies have reported a number of preoperative factors that increase the incidence or risk of SIRS in patients who underwent PCNL.

One study reported that female patients had a higher risk of SIRS after a PCNL, probably because of the greater propensity for urinary tract infections [16]. These results might be due to the short urethra, urethral proximity to the anus, intercourse-related trauma, or hormonal changes. On the other hand, several studies using multivariate analysis reported no definite correlation between sex and post-PCNL SIRS $[9,10,17]$. Recently, several prospective studies showed that cases of SIRS after a PCNL tend to involve females predominately [18-20]. Age and body mass index appear to have no definite correlation with the incidence of SIRS $[17,21,22]$
Diabetic PCNL patients have an independently greater risk of developing postoperative SIRS based on several studies [12,23,24], but a recent study showed no significant association between diabetes and SIRS [9]. Recent studies examined the relationship between diabetes and the immune system as well as why diabetes predisposes the patients to postoperative infections and major complications [25]. On the other hand, serum glucose or hemoglobin A1c levels may be more informative measurements because poor glucose control may be a greater contributing factor to infection than the diagnosis of diabetes itself [3]. Although there are no reports on the optimal glucose levels for PCNL procedures, the guidelines for sepsis care report a target glucose level of $\leq 180 \mathrm{mg} / \mathrm{dl}$ for ideal management [26]. Draga et al. [13] reported that patients with previous PCNL and paraplegia tend to have a higher incidence of SIRS after PCNL, but there are no prospective studies supporting these findings.

The elevation of white blood cells in the urine before PCNL was found to be associated with the development of SIRS [13]. These results are in agreement with prior studies using multivariate analysis $[9,17]$. The preoperative bladder urine culture was also shown to be related independently to post-PCNL SIRS in retrospective studies [9,10]. One prospective study reported that the rate of postoperative SIRS in patients with positive preoperative urine cultures was almost double that in patients with a sterile urine culture [27]; this result was in agreement with the findings in other prospective studies [14,18]. In addition, Patel et al. [28] reported a relatively high prevalence $30 \%)$ of multidrugresistant (MDR) bacteriuria in patients undergoing PCNL. Despite the appropriate use of preoperative antibiotics, MDR bacteriuria is a significant risk factor for postoperative infectious complications. For this reason, it is necessary

Table 1. Definitions of SIRS, infection, and sepsis

\begin{tabular}{ll}
\hline SIRS & Two or more of the following: \\
& - Body temperature $>38{ }^{\circ} \mathrm{C}$ or $<36{ }^{\circ} \mathrm{C}$ \\
& - Heart rate $>90 \mathrm{bpm}$ \\
& - Respiratory rate $>20$ breaths $/ \mathrm{min}$ or $\mathrm{PaCO}_{2}<32 \mathrm{mmHg}$ \\
& - White blood cell count $>12,000 \mathrm{cells} / \mu \mathrm{l}$ or $<4,000$ cells/ $\mu \mathrm{l}$ \\
Infection & Pathological process caused by the invasion of normally sterile tissue or fluid or the body cavity by pathogenic or potentially \\
& pathogenic micro-organisms \\
Sepsis & Clinical syndrome defined by the presence of both SIRS and infection \\
Severe sepsis & Sepsis resulting in organ dysfunction \\
Septic shock & Sepsis resulting in acute circulatory failure characterized by persistent arterial hypotension \\
\hline
\end{tabular}

SIRS: systemic inflammatory response syndrome, bpm: beats per minute.

Modified from the article of Levy et al. Intensive Care Med 2003;29:530-8 [11] with original copyright holder's permission. 
to complete the urine analysis and urine culture before the PCNL to evaluate the severity of the urinary tract infection and determine the appropriate antibiotic prophylaxis.

The laboratory findings of recent retrospective studies showed that independent factors, including the platelet-tolymphocyte ratio (PLR), neutrophil-to-lymphocyte ratio (NLCR), erythrocyte sedimentation rate (ESR) and C-reactive protein (CRP), affected the development of SIRS according to multivariate analysis $[15,29,30]$. These studies recommended close monitoring of PCNL patients with a PLR of $>114.1, \mathrm{NLCR} \geq 2.50, \mathrm{ESR}>6.5 \mathrm{~mm} / \mathrm{hr}$, and CRP $>0.65$ $\mathrm{mg} / \mathrm{dl}$ due to the possible infectious complications.

Previous retrospective studies have reported that the kidney stone burden is a risk factor for SIRS after PCNL $[7,17]$. During the surgical procedure, bacteria and endotoxins are released from the kidney stone. A larger kidney stone burden leads to a urinary obstruction with hydronephrosis and increases the difficulty of surgical procedures, which can prolong the operative time. Recent prospective studies confirmed these findings [13,14,21,31]. In addition, multivariate analysis found that a stone burden of $10 \mathrm{~cm}^{2}$ or greater was a significant predictor of postoperative SIRS [14]. The presence of preoperative hydronephrosis, which might be related to the kidney stone burden, was also found to be associated with post-PCNL SIRS [7,31]. On the other hand, there is a lack of consensus regarding the significance of preoperative hydronephrosis [3].

\section{Intraoperative Factors}

Several studies have reported the relationship between intraoperative multiple access tracts and operative time and SIRS after PCNL, which, as mentioned previously, could be influenced by the stone burden. Chen et al. [7], in a retrospective study, reported that multiple punctures and operative time are related to postoperative SIRS. Longer operative times are associated with higher postoperative SIRS rates because of the longer duration of high intra-pelvic pressures and the associated higher probability of pyelovenous backflow and irrigant absorption [8]. Several recent prospective studies have supported these results [10,21,32].

Intraoperative cultures are more important in predicting postoperative infections than preoperative cultures [8]. Retrospective studies, however, have limitations in their findings due to the lack of intraoperative culture results. A prospective cross-sectional study revealed a significant, tenfold increase in the risk of developing SIRS after PCNL in patients with positive stone cultures [33]. This may be due to bacterial colonization of stones and the release of endotoxins during the fragmentation and washing out of the stone fragments with irrigating fluid. Systemic absorption via the venous system or lymphatic channels can result from increased positive pressure during surgery [34]. Other prospective studies also reported evidence that a positive stone culture was associated with a higher risk of SIRS subsequent to surgery after adjusting for other predictors of SIRS $[18,27]$. In addition, positive intraoperative renal pelvis cultures have been indicated as a significant risk factor for the development of SIRS [14,35]. The main limitation of intraoperative cultures is that they are only available after the procedure and cannot be used to prevent the complications related to infection. Nevertheless, the results will assist in the optimal selection of antibiotic treatment in the event of SIRS after PCNL [5].

The effects of the stone composition on the incidence of postoperative SIRS has not been assessed directly [35]. Struvite and apatite stones likely carry a particularly high risk of infection after PCNL. One study showed that 94\% of the infected calculi were composed of struvite or apatite, and the levels of endotoxin in these stones were approximately 40 times higher than those in uninfected stones (represented by calcium oxalate, uric acid, and cysteine stones) [36].

\section{Postoperative Factors}

Degirmenci et al. [37], in a prospective study with PCNL patients, reported that the presence of residual stone fragments after PCNL is associated with the development of SIRS. This result appears to correspond with the findings of similar studies [19,38].

Several retrospective studies reported that the receipt of a blood transfusion is a key risk factor for the development of SIRS after PCNL [7,39]. A recent prospective study [21] reported that SIRS patients had a significantly higher blood transfusion requirement, as also reported in another prospective study [38]. Patients with complicated kidney stones and anatomic variations tend to have more injuries in the urinary tract. These could help facilitate bacterial inoculation in the disrupted renal parenchyma and translocation into the systemic circulation, resulting in subsequent infection [21]. 


\section{CONCLUSIONS}

Preventing and minimizing the risk of infectious complications is important for reducing the morbidity and mortality in patients after PCNL. Although serious infectious complications, such as sepsis and septic shock, are rare, easily overlooked symptoms could result in devastating postoperative mortality. Appropriate antibiotic prophylaxis and careful perioperative management in patients with the abovementioned risk factors will help decrease the incidence of infectious complications, including SIRS, after the PCNL. Owing to the lack of prospective studies and given the strict definitions of SIRS, larger, carefully designed, randomized controlled studies will be needed to confirm the independent perioperative risk factors for SIRS in patients with kidney stones undergoing PCNL.

\section{CONFLICT OF INTEREST}

No potential conflict of interest relevant to this article was reported.

\section{AUTHOR CONTRIBUTIONS}

H.S.C. participated in data collection and wrote the manuscript. S.I.J participated in the study design and coordination and helped to draft the manuscript. All authors read and approved the final manuscript.

\section{ORCID}

Ho Seok Chung, https://orcid.org/0000-0001-9883-1539 Seung Il Jung, https://orcid.org/0000-0003-4864-8175

\section{REFERENCES}

1. Fernstrom I, Johansson B. Percutaneous pyelolithotomy. A new extraction technique. Scand J Urol Nephrol 1976;10:257-9.

2. de la Rosette J, Assimos D, Desai M, Gutierrez J, Lingeman J, Scarpa R, et al.; CROES PCNL Study Group. The Clinical Research Office of the Endourological Society percutaneous nephrolithotomy global study: indications, complications, and outcomes in 5803 patients. J Endourol 2011;25:11-7.

3. Lai WS, Assimos D. Factors associated with postoperative infection after percutaneous nephrolithotomy. Rev Urol 2018; 20:7-11.
4. Deshmukh S, Sternberg K, Hernandez N, Eisner BH. Compliance with American Urological Association guidelines for post-percutaneous nephrolithotomy antibiotics does not appear to increase rates of infection. J Urol 2015;194:992-6.

5. Wollin DA, Joyce AD, Gupta M, Wong MYC, Laguna P, Gravas $S$, et al. Antibiotic use and the prevention and management of infectious complications in stone disease. World J Urol 2017; 35:1369-79.

6. Ivan SJ, Sindhwani P. Comparison of guideline recommendations for antimicrobial prophylaxis in urologic procedures: variability, lack of consensus, and contradictions. Int Urol Nephrol 2018;50:1923-37.

7. Chen L, Xu QQ, Li JX, Xiong LL, Wang XF, Huang XB. Systemic inflammatory response syndrome after percutaneous nephrolithotomy: an assessment of risk factors. Int J Urol 2008;15: 1025-8.

8. Singh P, Yadav S, Singh A, Saini AK, Kumar R, Seth A, et al. Systemic inflammatory response syndrome following percutaneous nephrolithotomy: assessment of risk factors and their impact on patient outcomes. Urol Int 2016;96:207-11.

9. He Z, Tang F, Lei H, Chen Y, Zeng G. Risk factors for systemic inflammatory response syndrome after percutaneous nephrolithotomy. Prog Urol 2018;28:582-7.

10. Erdil T, Bostanci Y, Ozden E, Atac F, Yakupoglu YK, Yilmaz AF, et al. Risk factors for systemic inflammatory response syndrome following percutaneous nephrolithotomy. Urolithiasis 2013; 41:395-401.

11. Levy MM, Fink MP, Marshall JC, Abraham E, Angus D, Cook D, et al. $2001 \mathrm{SCCM} / \mathrm{ESICM} / \mathrm{ACCP} / \mathrm{ATS} / \mathrm{SIS}$ International Sepsis Definitions Conference. Intensive Care Med 2003;29:530-8.

12. Wei W, Leng J, Shao H, Wang W. Diabetes, a risk factor for both infectious and major complications after percutaneous nephrolithotomy. Int J Clin Exp Med 2015;8:16620-6.

13. Draga RO, Kok ET, Sorel MR, Bosch RJ, Lock TM. Percutaneous nephrolithotomy: factors associated with fever after the first postoperative day and systemic inflammatory response syndrome. J Endourol 2009;23:921-7.

14. Korets R, Graversen JA, Kates M, Mues AC, Gupta M. Postpercutaneous nephrolithotomy systemic inflammatory response: a prospective analysis of preoperative urine, renal pelvic urine and stone cultures. J Urol 2011;186:1899-903.

15. Cetinkaya M, Buldu I, Kurt O, Inan R. Platelet-to-lymphocyte ratio: a new factor for predicting systemic inflammatory response syndrome after percutaneous nephrolithotomy. Urol J 2017;14:4089-93.

16. Sharifi Aghdas $F$, Akhavizadegan $H$, Aryanpoor $A$, Inanloo $H$, Karbakhsh M. Fever after percutaneous nephrolithotomy: contributing factors. Surg Infect (Larchmt) 2006;7:367-71.

17. Yang $\mathrm{T}$, Liu $\mathrm{S}, \mathrm{Hu} J$, Wang $\mathrm{L}$, Jiang $\mathrm{H}$. The evaluation of risk factors for postoperative infectious complications after percutaneous nephrolithotomy. Biomed Res Int 2017;2017: 4832051. 
18. Mariappan P, Smith G, Bariol SV, Moussa SA, Tolley DA. Stone and pelvic urine culture and sensitivity are better than bladder urine as predictors of urosepsis following percutaneous nephrolithotomy: a prospective clinical study. J Urol 2005;173: 1610-4.

19. Walton-Diaz A, Vinay JI, Barahona J, Daels P, Gonzalez $M$, Hidalgo JP, et al. Concordance of renal stone culture: PMUC, RPUC, RSC and post-PCNL sepsis-a non-randomized prospective observation cohort study. Int Urol Nephrol 2017;49: 31-5.

20. Chew BH, Miller NL, Abbott JE, Lange D, Humphreys MR, Pais VM Jr, et al. A randomized controlled trial of preoperative prophylactic antibiotics prior to percutaneous nephrolithotomy in a low infectious risk population: a report from the EDGE consortium. J Urol 2018;200:801-8.

21. Singh I, Shah S, Gupta S, Singh NP. Efficacy of intraoperative renal stone culture in predicting postpercutaneous nephrolithotomy urosepsis/systemic inflammatory response syndrome: a prospective analytical study with review of literature. J Endourol 2019;33:84-92.

22. Rivera M, Viers B, Cockerill P, Agarwal D, Mehta R, Krambeck A. Pre- and postoperative predictors of infection-related complications in patients undergoing percutaneous nephrolithotomy. J Endourol 2016;30:982-6.

23. Ronald A, Ludwig E. Urinary tract infections in adults with diabetes. Int J Antimicrob Agents 2001;17:287-92.

24. Gutierrez J, Smith A, Geavlete P, Shah H, Kural AR, de Sio M, et al. Urinary tract infections and post-operative fever in percutaneous nephrolithotomy. World J Urol 2013;31:1135-40.

25. Giubilato S, Liuzzo G, Brugaletta S, Pitocco D, Graziani F, Smaldone C, et al. Expansion of CD4+CD28null T-lymphocytes in diabetic patients: exploring new pathogenetic mechanisms of increased cardiovascular risk in diabetes mellitus. Eur Heart J 2011;32:1214-26.

26. Dellinger RP, Levy MM, Rhodes A, Annane D, Gerlach H, Opal $\mathrm{SM}$, et al.; Surviving Sepsis Campaign Guidelines Committee including the Pediatric Subgroup. Surviving Sepsis Campaign: international guidelines for management of severe sepsis and septic shock, 2012. Intensive Care Med 2013;39:165-228.

27. Margel D, Ehrlich Y, Brown N, Lask D, Livne PM, Lifshitz DA. Clinical implication of routine stone culture in percutaneous nephrolithotomy--a prospective study. Urology 2006;67:26-9.

28. Patel N, Shi W, Liss M, Raheem O, Wenzler D, Schallhorn C, et al. Multidrug resistant bacteriuria before percutaneous nephrolithotomy predicts for postoperative infectious complications.
J Endourol 2015;29:531-6.

29. Sen V, Bozkurt IH, Aydogdu O, Yonguc T, Yarimoglu S, Sen P, et al. Significance of preoperative neutrophil-lymphocyte count ratio on predicting postoperative sepsis after percutaneous nephrolithotomy. Kaohsiung J Med Sci 2016;32:507-13.

30. Ganesan V, Brown RD, Jimenez JA, De S, Monga M. C-reactive protein and erythrocyte sedimentation rate predict systemic inflammatory response syndrome after percutaneous nephrolithotomy. J Endourol 2017;31:638-44.

31. Mariappan P, Smith G, Moussa SA, Tolley DA. One week of ciprofloxacin before percutaneous nephrolithotomy significantly reduces upper tract infection and urosepsis: a prospective controlled study. BJU Int 2006;98:1075-9.

32. Omar M, Noble M, Sivalingam S, El Mahdy A, Gamal A, Farag $M$, et al. Systemic inflammatory response syndrome after percutaneous nephrolithotomy: a randomized single-blind clinical trial evaluating the impact of irrigation pressure. J Urol 2016;196:109-14.

33. Roushani A, Falahatkar S, Sharifi SH, Mahfoozi L, Saadat SM, Allahkhah $A$, et al. Intra-operative stone culture as an independent predictor of systemic inflammatory response syndrome after percutaneous nephrolithotomy. Urolithiasis 2014;42:455-9.

34. Lojanapiwat B, Kitirattrakarn P. Role of preoperative and intraoperative factors in mediating infection complication following percutaneous nephrolithotomy. Urol Int 2011;86:44852.

35. Kreydin El, Eisner BH. Risk factors for sepsis after percutaneous renal stone surgery. Nat Rev Urol 2013;10:598-605.

36. McAleer IM, Kaplan GW, Bradley JS, Carroll SF, Griffith DP. Endotoxin content in renal calculi. J Urol 2003;169:1813-4.

37. Degirmenci T, Bozkurt IH, Celik S, Yarimoglu S, Basmaci I, Sefik E. Does leaving residual fragments after percutaneous nephrolithotomy in patients with positive stone culture and/or renal pelvic urine culture increase the risk of infectious complications? Urolithiasis 2018 Jun 4 [Epub]. DOI: 10.1007/s00240018-1063-9.

38. Ramaraju K, Paranjothi AK, Namperumalsamy DB, Chennakrishnan I. Predictors of systemic inflammatory response syndrome following percutaneous nephrolithotomy. Urol Ann 2016;8:449-53.

39. Shoshany O, Margel D, Finz C, Ben-Yehuda O, Livne PM, Holand $\mathrm{R}$, et al. Percutaneous nephrolithotomy for infection stones: what is the risk for postoperative sepsis? A retrospective cohort study. Urolithiasis 2015;43:237-42. 